\title{
Erratum to: Test-retest reliability of the underlying latent factor structure of alcohol subjective response
}

Joseph A. Lutz ${ }^{1}$ Emma Childs ${ }^{1}$

Published online: 16 February 2017

(C) Springer-Verlag Berlin Heidelberg 2017

Erratum to: Psychopharmacology

DOI:10.1007/s00213-017-4535-7

The original version of this article, published on 27 January 2017, unfortunately contained an error in the values in the final three rows of Table 2. Correct table is presented here.

The online version of the original article can be found at http://dx.doi.org/ 10.1007/s00213-017-4535-7.

Emma Childs

echilds@psych.uic.edu

1 Department of Psychiatry, University of Illinois at Chicago, 1601W.

Taylor St, Chicago, IL 60612, USA 
Table 2 Exploratory factor analysis solution for all $\mathrm{AUC}_{\mathrm{NET}}$ values $(\mathrm{N}=312)$.

\begin{tabular}{lllll}
\hline & $\begin{array}{l}\text { Factor } 1 \\
\text { Positive mood }\end{array}$ & $\begin{array}{l}\text { Factor } 2 \\
\text { Drug effects and Urge }\end{array}$ & $\begin{array}{l}\text { Factor 3 } \\
\text { Stimulation/ Euphoria }\end{array}$ & $\begin{array}{l}\text { Factor 4 } \\
\text { Sedation }\end{array}$ \\
\hline POMS Elation &. $\mathbf{7 6 6}$ & .007 & -.022 & -.032 \\
POMS Vigour &. $\mathbf{7 3 4}$ & -.009 & -.032 & -.160 \\
POMS Friendliness & $\mathbf{. 6 5 5}$ & -.026 & .004 & .073 \\
BAES Stimulation &. $\mathbf{5 4 1}$ & .039 & .201 & -.112 \\
DEQ Like & .023 & $\mathbf{. 8 0 1}$ & .047 & -.107 \\
DEQ More & -.032 &. $\mathbf{7 2 1}$ & -.097 & -.207 \\
DEQ Feel & -.005 &. $\mathbf{6 3 8}$ & .054 & .237 \\
DEQ High & .017 & $\mathbf{. 5 2 6}$ & .059 & .174 \\
ARCI-A & .027 & .019 & $\mathbf{9 3 7}$ & .071 \\
ARCI-BG & -.094 & -.088 & $\mathbf{6 8 0}$ & -.425 \\
ARCI-MBG & .220 & .105 & $\mathbf{5 7 1}$ & .051 \\
BAES Sedation & -.054 & .003 & .021 &. $\mathbf{7 6 7}$ \\
ARCI-PCAG & -.103 & .084 & -.170 & .722 \\
POMS Fatigue & -.130 & -.048 & -.016 &. $\mathbf{5 9 7}$ \\
Eigenvalues & 4.451 & 2.555 & 1.358 & 1.227 \\
\% of variance & 31.7 & 18.2 & 9.7 & 8.7 \\
$\alpha$ & .805 & .763 & .807 & .802 \\
\hline
\end{tabular}

Note: Scales with factor loadings $<0.4$ were omitted. ARCI: Addiction Research Center Inventory (A=Amphetamine; $\mathrm{MBG}=$ Morphine-Benzedrine Group; $\mathrm{BG}=$ Benzedrine Group; PCAG=PentobarbitalClorpromazine-Alcohol Group); BAES: Biphasic Alcohol Effects Scale; POMS: Profile Of Mood States; DEQ: Drug Effects Questionnaire 